\title{
Effect of Photoperiod on the Development of Wheel-Running Activity Rhythms in LEW/Ztm Rats
}

\author{
URSULA SIEBERT ${ }^{1}$ AND FRANZISKA WOLLNIK \\ Department of Biology, University of Konstanz, P.O. Box 5560, D-7750 Konstanz, Germany
}

Received 24 April 1992

\begin{abstract}
SIEBERT, U. AND F. WOLLNIK. Effect of photoperiod on the development of wheel-running activity rhythms in LEW/Ztm rats. PHYSIOL BEHAV 53(6) 1145-1150, 1993.-Wheel-running activity of LEW/Ztm rats is characterized by a multimodal pattern consisting of two activity bouts about $3-5 \mathrm{~h}$ apart. In this study we investigated the development of activity rhythms in LEW rats born and raised under three different photoperiods (LD 18:6, LD 12:12, and LD 6:18). Wheel-running activity was measured for 6 weeks in LD and for another 6 weeks in constant darkness (DD). The length of the photoperiod influenced the phase relationship between the two activity bouts only during the first week after weaning. Then, the characteristic activity pattern was established independently of the length of the photoperiod. However, development under long photoperiods (LD 18:6) resulted in a temporary increase in the level of activity and a significant shortening of the free-running period under DD. These results indicate that the multimodal activity pattern displayed by LEW rats is controlled by separate activity oscillators that establish their unique phase relationship early during development.
\end{abstract}

Circadian and ultradian rhythms Development Multioscillatory system Activity feedback

DAILY rhythms of various behavioral and physiological parameters in the rat appear at different developmental stages $(3,13)$. The earliest functional rhythm is the daily rhythm in the pineal content of the enzyme $\mathrm{N}$-acetyltransferase, which appears at postnatal day 4 (7). Behavioral rhythms such as locomotor activity do not emerge until postnatal day $20(3,16)$ and appear to go through a process of maturation. At first, ultradian rhythms are more prominent, then a circadian pattern gradually emerges (6). It has been suggested that the gradual development of behavioral circadian rhythms reflects a strengthening in the coupling of multiple oscillatory units both with each other and with various afferent and efferent pathways $(3,8,9)$.

The purpose of the present study was to examine the development of circadian activity rhythms in a specific inbred strain of laboratory rats, LEW/Ztm. Rats of this strain typically show a rather unusual multimodal activity pattern with two or three activity bouts about $3-5 \mathrm{~h}$ apart (19). This activity pattern generates ultradian peaks in the range of 4 and $4.8 \mathrm{~h}$ when tested using methods of period analysis. Previous studies indicated that these ultradian components are not caused by a specific ultradian pacemaker (20), but may instead reflect a distinct coupling of multiple circadian oscillators (19). An earlier study (15) indicated that in 3-6-week-old rats, the phase relationship between individual activity bouts may be modified by light-dark (LD) cycles of different photoperiods. In adult animals, however, the typical multimodal pattern was not affected by the changing photoperiods, suggesting that the characteristic coupling of the multiple oscillatory units cannot be changed once it has been established.

In this study we investigated the development of the wheelrunning activity rhythms of LEW rats born and raised under three different photoperiods in order to examine how ultradian and circadian rhythms interact during ontogeny and how different LD cycles influence the characteristic activity pattern of LEW rats.

\section{METHOD}

\section{Animals and Housing}

Male rats of the inbred strain LEW/Ztm, originally obtained from the Central Animal Laboratory at the Hanover Medical School (FRG) were born and raised under three different photoperiods:

1. LD 18:6 (18 h of light and $6 \mathrm{~h}$ of darkness; $n=4)$,

2. LD 12:12 $(n=4)$,

3. LD $6: 18(n=4)$.

At the age of 21 days the animals were weaned and placed in individual cages (Makrolon Type IV, $35 \times 55 \times 20 \mathrm{~cm}$ ) with

\footnotetext{
' Requests for reprints should be addressed to Ursula Siebert, Fakultät für Biologie, Universität Konstanz, Postfach 5560, D-7750 Konstanz, Germany.
} 

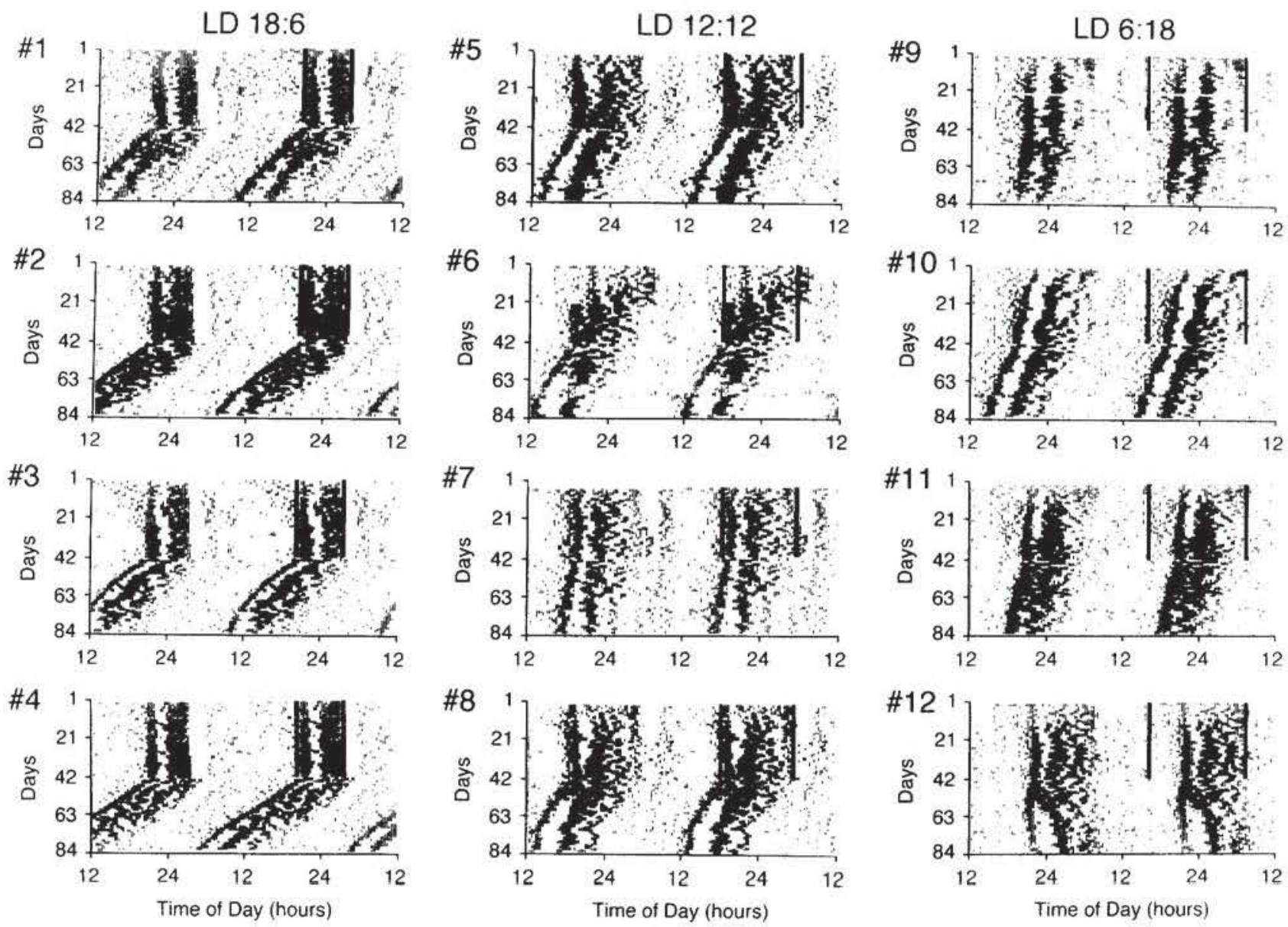

FIG. 1. Double-plotted wheel-running activity records of 12 LEW rats kept under three different photoperiods: LD 18:6 (left), LD 12:12 (center). and LD 6:18 (right). Numbers on the vertical axis denote days of experiment, numbers on the horizontal axis denote daytime hours. The black bars on the right side of each plot indicate beginning and end of the dark period.

constant access to food, water, and a running wheel (diameter $35 \mathrm{~cm}$, width $10 \mathrm{~cm}$ ). Spontaneous wheel-running activity was measured for a total of 12 weeks. After 6 weeks under LD entrainment, all animals were exposed to continuous darkness (DD) for another 6 weeks. Cages were cleaned every 4 weeks, and water and food was checked 2-3 times a week at random times during the day.

\section{Data Collection and Analysis}

Three magnetic reed switches were attached to the axle of each running wheel so that one complete wheel revolution resulted in three impulses. These impulses were read online by a microcomputer and stored on disk every $5 \mathrm{~min}$. All subsequent calculations were based on these 5-min counts. Event records were visualized as double plots with a resolution of $20 \mathrm{~min}$. Maximum height of a 20 -min bin indicates more than 200 counts (i.e., an average of 10 counts/min), while a blank space indicates fewer than 20 counts (i.e., an average of 1 count $/ \mathrm{min}$ ).

The circadian period $\tau$ of each animal was calculated both weekly and for longer intervals of 3 weeks using the chi square periodogram (17). Furthermore, the harmonic spectral analysis $(5,11)$ was used to verify the presence of periodic components.
The results of both methods were always compared to verify the reliability of the analyses. Further details of the statistical models underlying these two approaches are described elsewhere (19).

Additional parameters calculated from the activity recordings include:

1. amount of activity: the total number of impulses within a 24-h period, given as impulses/day;

2. amount of activity during the light and dark phase, given in percent.

Furthermore, weekly data were averaged within animals and smoothened using a moving average over $35 \mathrm{~min}$. The resulting daily profiles were used to determine times of activity onset and offset for each animal. Onset was determined as the start time of the first block of at least six consecutive 5-min intervals that contained more than five impulses and was not separated from the next block of activity by more than $30 \mathrm{~min}$. Offset was determined in an analogous way. Activity peaks were defined as bins with maximal activity. Activity time $\alpha$, i.e., time between onset and offset, and the phase difference $\Psi_{1-2}$ between the two activity peaks were calculated from these values.

Differences between groups and between lighting conditions were assessed using standard procedures of variance analysis 

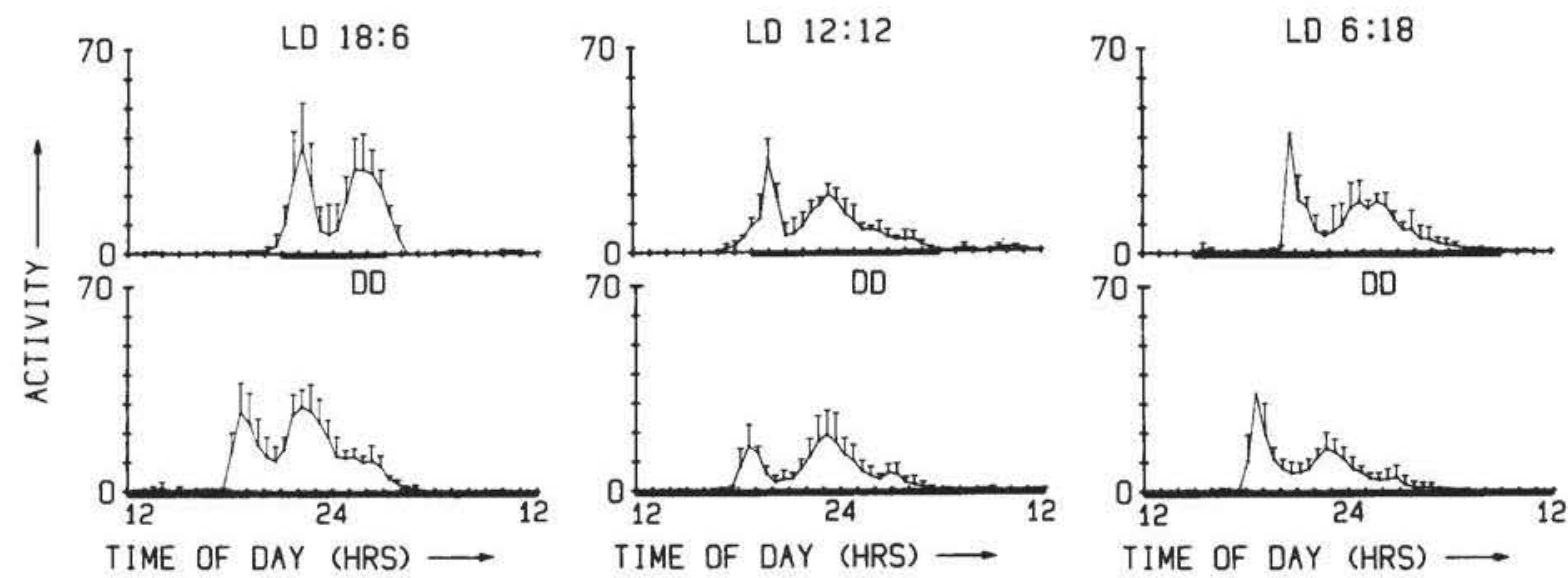

FIG. 2. Average daily profiles of wheel-running activity under three different photoperiods (upper panel) and subsequent DD (lower panel). Thirty-minute mean values are plotted over time, with standard errors between animals shown as vertical lines. Daily profiles of free-running animals are plotted as a function of circadian time based on the $\tau$ values revealed by periodogram analysis.

(ANOVA, SAS). Averaged data are reported as means \pm standard error (SEM). Post hoc comparisons were made using multiple $t$-tests. Spearman's correlation analysis was applied to compare values of period length $\tau$ and activity level.

\section{RESULTS}

Activity records of all animals are shown in Fig. 1. With more than $60 \%$ of the activity occurring during the dark phase, the animals were nocturnal from the day of weaning. Periodogram analyses showed a significant 24 -h rhythm from the third week on. All animals exhibited the typical activity pattern of LEW rats with two short activity bouts about 3-5 h apart from each other. Animals kept under LD 12:12 or LD 6:18 showed an additional weaker bout of activity at the end of the dark phase. This third activity bout was most pronounced during the first week of the experiment and occurred directly before the dark-light transition. During exposure to the subsequent continuous darkness the animals showed a free-running period $\tau$ shorter than $24 \mathrm{~h}$. ANOVA revealed a significant difference in $\tau$ between the three groups, $F(2,11)=11.02, p \leq 0.01$. Animals that had previously been kept in a long photoperiod (LD 18:6) had a significantly shorter period $(\tau=23.69 \pm 0.05 \mathrm{~h})$ than animals kept in LD 12:12 ( $\tau=23.89 \pm 0.04 \mathrm{~h})$ or LD 6:18 ( $\tau$ $=23.93 \pm 0.02 \mathrm{~h}$ ).

Figure 2 depicts average daily profiles for each group during the entrained state (upper panel) and under DD (lower panel). The two activity bouts were most pronounced in animals entrained to LD 18:6. Under DD the activity profiles of all three groups showed a smaller third bout at the end of activity. Characteristic frequency components for each group and each lighting condition were analyzed using two different methods of period analysis, i.e., the harmonic spectral analysis and the periodogram. According to both methods, all three groups showed a clear circadian component and additional ultradian components in the range of 3 to $4.8 \mathrm{~h}$. A more detailed analysis of spectral estimates during DD did not show any significant effect of early photoperiodic exposure on the amplitudes of either circadian or ultradian rhythmic components.

Figure 3 shows developmental changes in the level of activity, the activity time $\alpha$, and the phase difference $\Psi_{1-2}$ during the 12 weeks of the experiment. In all three groups, the activity level increased continuously during the first 5 weeks. After week 5 , both the LD 12:12 and LD 6:18 group showed a steady decline in their activity level. Animals kept under LD 18:6, however, increased their activity level during the first 3 weeks under DD. During the last 3 weeks under DD, the activity level of the LD 18:6 group declined, finally reaching values not different from those of the other two groups. Activity time $\alpha$ of the LD 18:6 group showed a similar increase and decrease under DD conditions. The phase difference $\Psi_{1-2}$ between the two activity peaks differed between weeks of experiment, $F(11,23)=4.63, p \leq$ 0.01 , but not between groups, $F(2,23)=1.05$, NS. The mean $\Psi_{1-2}$ of all three groups was $3.72 \pm 0.08 \mathrm{~h}$. Only during the first week of the experiment, $\Psi_{1-2}$ was higher in animals kept under LD 12:12 (5.5 $\pm 0.66 \mathrm{~h})$ and LD 6:18 (5.4 $\pm 0.8 \mathrm{~h})$. This difference disappeared during the following weeks. $\Psi_{1-2}$ of all animals decreased during the 6 weeks of entrainment and increased again under free-running conditions. As a result, the two activity peaks were significantly further apart from each other during week 12 $(4.08 \pm 0.13 \mathrm{~h})$ than during week $6(2.88 \pm 0.18 \mathrm{~h})$.

Statistical analyses (two-way ANOVA) of the activity level, activity time $\alpha$, and the phase difference $\Psi_{1-2}$ between groups (LD 18:6; LD 12:12; LD 6:18) and lighting conditions (LD; DD) revealed significant effects of groups and group $\times$ lighting interactions for the level of activity, $F_{\text {groups }}(2,23)=10.39, p \leq$ $0.01 ; F_{\text {interactions }}(22,23)=8.08, p \leq 0.01$, and activity time $\alpha$, $F_{\text {groups }}(2,23)=5.44, p \leq 0.01 ; F_{\text {interactions }}(22,23)=9.42, p \leq$ 0.01 . During the entrained state, rats of the LD 12:12 group displayed the longest activity time $(\alpha=8.24 \pm 0.32 \mathrm{~h})$. Rats kept in LD 18:6 showed a significant increase in the level of activity and activity time $\alpha$ when they were transferred to DD $(p \leq 0.01)$. In DD, this group had a significantly higher activity level ( $2104 \pm 882$ impulses/day) and activity time $\alpha(8.1 \pm 1.5$ h) than the other two groups $(p \leq 0.01)$. There were no differences in $\Psi_{1-2}$ between groups or lighting conditions.

Comparison of group means suggested that differences in the free-running period under DD may be related to differences in the activity level. Indeed, correlation analysis using mean values of weeks 7-12 for each animal demonstrated a small but significant correlation $\left(r_{\mathrm{s}}=-0.67, p \leq 0.05\right)$ between the free- 

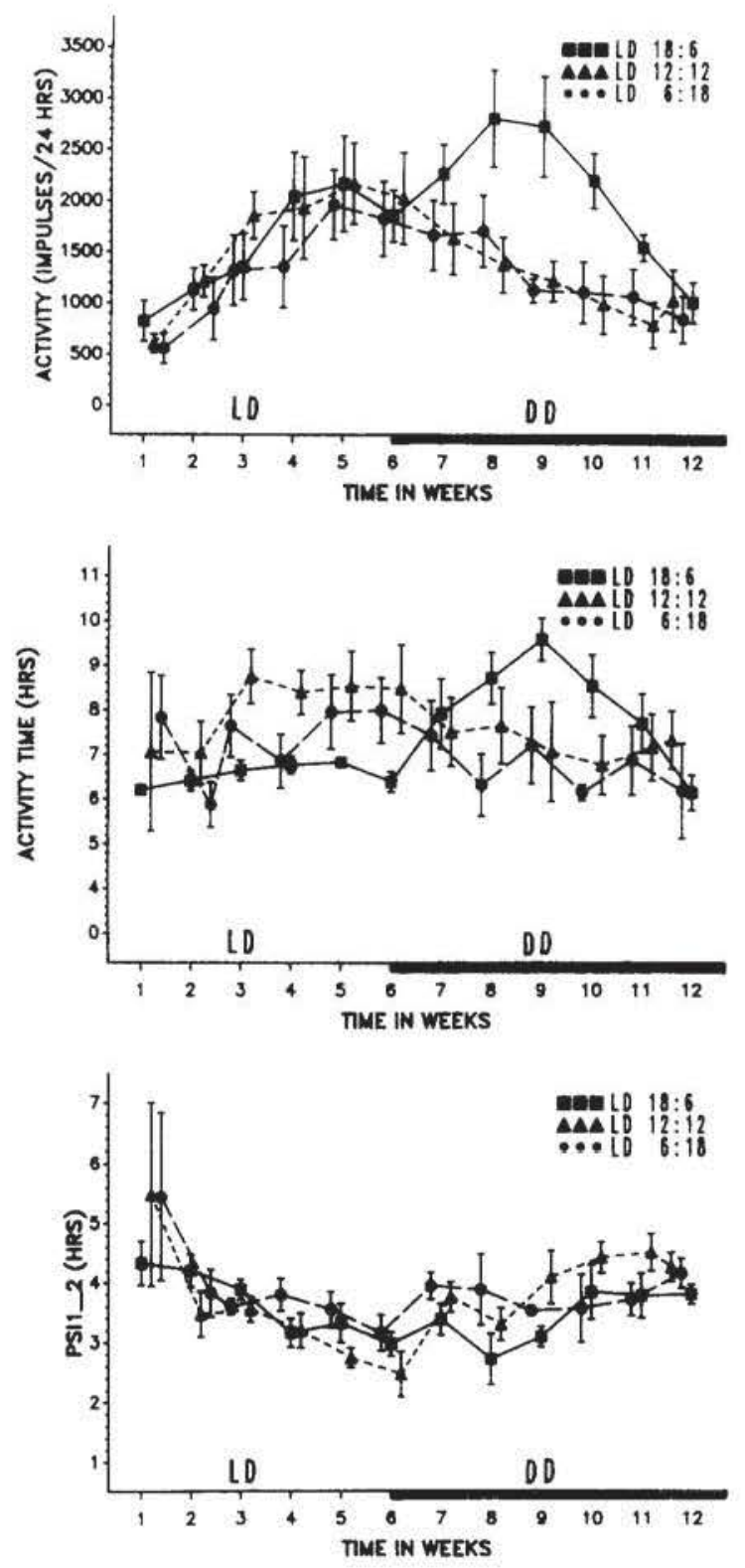

FIG. 3. Changes in the level of activity (defined as total number of impulses per day, upper panel), activity time (defined as the time between activity onset and offset, middle panel), and phase difference between the two activity peaks $\left(\Psi_{1-2}\right.$. lower panel) for the three different groups (LD 18:6, LD 12:12, LD 6:18). Weekly mean values are plotted over time, with standard errors between animals shown as vertical lines. Animals were kept in LD for the first 6 weeks after weaning and in DD during weeks $6-12$.

running period and the level of activity (Fig. 4). However, when tested on a weekly basis, this correlation was only significant during week 9 , when differences in the level of activity were most pronounced $\left(r_{\mathrm{s}}=-0.65, p \leq 0.05\right)$. By week 12 , differences in the activity level had completely disappeared, while differences in period length were still significant. Furthermore, within the LD 18:6 group, changes in activity level were not correlated with changes in period length $\left(r_{\mathrm{s}}=-0.03 ; \mathrm{NS}\right)$.
DISC USSION

The present results demonstrate that early photoperiodic exposure has a distinct effect on the development of circadian activity rhythms, but only a minor effect on the characteristic ultradian activity components of LEW rats.

It has been suggested that the gradual development of behavioral circadian rhythms reflects the postnatal maturation of the circadian system located in the SCN (3.8.9). Animals of all three groups showed a clear day-night difference in their wheelrunning activity during the first week after weaning that was independent of the photoperiod they were kept in. These results confirm previous findings demonstrating a circadian rhythm in locomotor activity in 21-day-old rats under L.D 12:12 entrainment or DD $(2,6,16)$.

Differences in the activity pattern between the three photoperiodic treatments were most pronounced during the first week after weaning (week 1) when activity was clustered around the LD transitions in all three groups under investigation (see Fig. 1. days $1-7$ ). Therefore, activity time $\alpha$ was longer in animals raised under LD 6:18 than in animals raised under LD 12:12 or LD 18:6, and the activity bouts lay further apart (Fig. 3. bottom panel, first week). LEW rats exposed to running wheels at $60-70$ days of age did not show an increased phase relationship between the two peaks (19). Therefore, the increased phase relationship between the two activity bouts seems to be an agerelated phenomenon rather than a general effect of initial wheel exposure. The increased phase relationship between the two activity bouts disappeared during the following 5 weeks under LD entrainment, and all rats showed the typical activity pattern of the LEW strain with two activity bouts about $3.5 \mathrm{~h}$ apart from each other.

After transfer into DD, differences between the three groups were found for the free-running period. the level of activity, and the activity time $\alpha$. The most obvious effect was a rather short free-running period in animals born and raised under LD 18:6 compared to animals raised under LD 12:12 or under LD 6:18, suggesting that the intrinsic mechanism of the circadian system had been affected by the previous photoperiodic treatment. Because all animals of a group were taken from the same litter, we

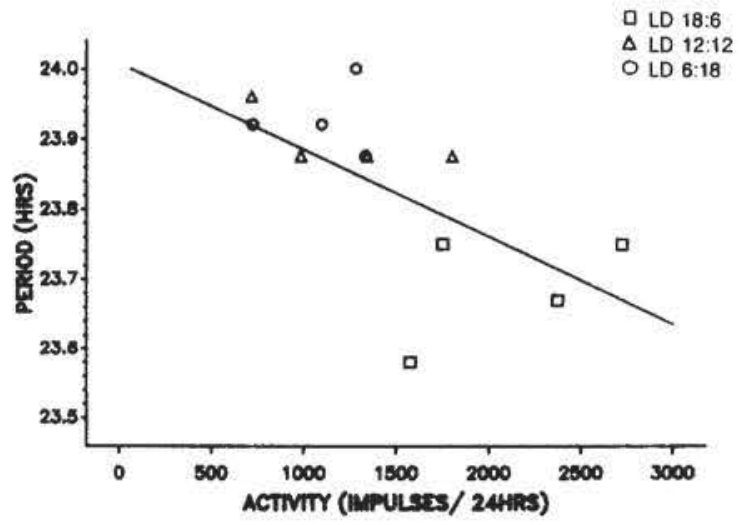

FIG. 4. Relationship between free-running period and level of activity. For each animal, the mean period length during weeks $7-12$ was plotted against the amount of activity, given as number of impulses per day. Correlation factor $r_{\mathrm{s}}=-0.67(p \leq 0.05)$. 
cannot completely rule out the possibility that the observed differences were merely due to maternal or genetic effects. However, the LEW strain has been bred for many generations under a strong inbreeding regime, and animals from different litters must be considered genetically identical. In addition, analysis of many different litters born and raised under LD 12:12 did not show significant differences in free-running period between litters. These results support our view that the observed differences are due to the photoperiodic treatment rather than to other, unknown differences between litters.

It has long been accepted that the circadian system of mammals consists of multiple circadian oscillators coordinated by both hierarchical and nonhierarchical coupling relationships (14). Previous studies of the LEW strain suggested that its unusual activity pattern may be caused by a distinct coupling of multiple circadian oscillators $(15,19,20)$. This hypothesis is supported by the present study which found differences in the phase relationship between the major activity bouts in very young animals, and by the finding that the phase relationship between the two activity bouts increased during DD (Fig. 3). However, maturation of the circadian system under different photoperiods did not result in any long-lasting modification of the activity pattern. Previous experiments with adult LEW rats also showed that the temporal relationship of the major activity bouts is not altered by changing photoperiod (15). These results suggest that the phase relationship between the oscillatory units remains constant once a clear activity rhythm has been established.

An important result of the present study is the difference of the free-running period $\tau$ between groups. The rather short freerunning period of rats born and raised under LD 18:6 can be explained by the two-oscillator model proposed by Pittendrigh and Daan (12) as an aftereffect of the photoperiodic treatment. According to this model, the circadian system consists of two major oscillators which are coupled to lights-off and lights-on as well as to each other. Entrainment to a rather long photoperiod, e.g., LD 18:6, would reduce the phase difference between these two oscillators and thereby shorten the activity time $\alpha$. Furthermore, the model assumes that the free-running period of the compound system is a function of the period lengths of the two constituent oscillators and their mutual coupling strength. Because the coupling strength depends on the phase difference between the two oscillators, it can be modified by the prior photoperiod. According to the model, long photoperiods change the mutual coupling strength so that the oscillator with the shorter period (i.e., the evening oscillator) exerts a stronger influence on the second oscillator (i.e., the morning oscillator). A short photoperiod would thus lead to a shorter $\alpha$ and shorter $\tau$ in subsequent freerun.

In light of recent observations that behavioral arousal may produce feedback effects on the circadian system $(10,18)$, changes in the free-running period may also be explained by alterations of the activity level. Although a relationship between period length and activity has been recognized many years ago (1), activity-dependent influences on the endogenous circadian system have only recently been acknowledged. For example, access to a running wheel has been shown to increase the level of activity and to shorten the free-running period in rats (21). A negative correlation between the number of wheel revolutions and the free-running period has also been observed in mice (4). In accordance with this observation, the present study demonstrated that rats kept under a long photoperiod (LD 18:6) had an increased activity level when transferred into DD and a significantly shorter free-running period than rats kept under LD 12:12 or LD 6:18. Correlation analysis (Fig. 4) supported the notion that the free-running period during DD was related to the level of activity. The finding that differences in period length were still present during week 12 , when differences in activity levels had disappeared, does not necessarily invalidate the activity feedback hypothesis. It may well be that the increase of activity induced a longer-lasting effect on the free-running period. However, a longer registration period would be necessary to confirm this hypothesis.

In conclusion, the present study supports the hypothesis that the unusual multimodal activity pattern of the LEW strain reflects a unique phase relationship between multiple circadian oscillators. However, the coupling between these oscillatory units is rather tight and is not affected by LD cycles of different photoperiods in both adult animals and young animals during maturation of the circadian system. Nevertheless, photoperiodic treatment during the early neonatal life clearly affects the circadian system. Development under long photoperiods (LD 18: 6) resulted in a shorter free-running period and in an increased activity level. The strain LEW may, thus, be a good animal model to further explore activity related effects on the circadian system.

\section{ACKNOWLEDGEMENT}

This research was supported by a grant from Deutsche Forschungsgemeinschaft (Wo-354/3-2).

\section{REFERENCES}

1. Aschoff, J. Exogenous and endogenous components in circadian rhythms. Cold Spring Harb. Symp. Quant. Biol. 25:11-28; 1960.

2. Cambras, T.; Diez-Noguera, A. Evolution of rat motor activity circadian rhythm under three different light patterns. Physiol. Behav. 49(1):63-68; 1991.

3. Davis, F. C. Ontogeny of circadian rhythms. In: Aschoff, J., ed. Handbook of behavioral neurobiology, vol. 4. Biological rhythms. New York: Plenum Press; 1981:257-274.

4. Edgar, D.; Connie, E.; Dement, W. Activity feedback to the mammalian circadian pacemaker: Influence on observed measures of rhythm period length. J. Biol. Rhythms 6(3):185-199; 1991.

5. Halberg, F.; Panofsky, H. I. Thermo-variance spectra; Methods and clinical illustrations. Exp. Med. Surg. 19:285-309; 1961.

6. Honma, S.; Honma K. Interaction between circadian and ultradian rhythms of spontaneous locomotor activity in rats during the early developmental period. In: Schulz, H.; Lavie, P., eds. Ultradian rhythms in physiology and behavior. Berlin: Springer Verlag; 1985: 95-109.
7. Klein, D. C.; Weller, J. L. Indole metabolism in the mammalian pineal gland. In: Reiter, R. J., ed., The pineal gland. Boca Raton: CRC Press; 1981:199-227.

8. Moore, R. Y. The suprachiasmatic nucleus and the organization of a circadian system. Trends Neurosci. 5:404-407; 1982.

9. Moore, R. Y.; Bernstein, M. E. Synaptogenesis in the rat suprachiasmatic nucleus demonstrated by electron microscopy and synapsin I immunoreactivity. J. Neurosci. 9(6):2151-2162; 1989.

10. Mrosovsky, N.; Reebs, S.; Honrado, G.; Salmon, P. Behavioural entrainment of circadian rhythms. Experientia 45:696-702; 1989.

11. Panovsky, H.; Halberg, F., II. Thermo-variance spectra; Simplified computational example and other methodology. Exp. Med. Surg. 19:322-338; 1961.

12. Pittendrigh, C; Daan, S. A functional analysis of circadian pacemakers in nocturnal rodents V. Pacemaker structure: A clock for all seasons. J. Comp. Physiol. 106:333-355; 1976.

13. Reppert, S. M. Maternal entrainment of the developing circadian system. Ann. NY Acad. Sci. 453:162-169; 1985. 
14. Rosenwasser, A. M.; Adler, N. T. Structure and function in circadian timing systems: Evidence for multiple coupled circadian oscillators Neurosci. Biobehav, Rev, 10:431-448: 1986.

15. Siebert, U.: Wollnik. F. Wheel-running activity rhythms in two inbred strains of laboratory rats under different photoperiods. Physiol. Behav. 50(6):1137-1143:1991.

16. Smith, G. K.: Anderson, V. Effects of maternal isolation on development of activity rhythms in infant rats. Physiol. Behav. 33:751756: 1984 .

17. Sokolove, P. G.; Bushell, W. N. The chi square periodogram: Its utility for analysis of circadian rhythms. J. Theor. Biol. 72:131-160: 1978.
18. Turek, F. Effects of stimulated physical activity on the circadian pacemaker of vertebrates. J. Biol. Rhythms 4(2):135-147: 1989.

19. Wollnik. F. Strain differences in the pattern and intensity of wheel running activity in laboratory rats. Experientia 47:593598: 1991.

20. Wollnik. F: Turek. F. SCN lesions abolish ultradian and circadian components of activity rhythms in LEW/Ztm rats. Am. J. Physiol. 256:RI027-R1039: 1989.

21. Yamada, N.: Shimada. K.: Takahashi, K.: Takahashi, S. Relationship between free-running period and motor activity in blinded rats. Brain Res. Bull. 25:115-119: 1990. 